\title{
Prevalência de ausência de função dos tendões do músculo flexor superficial do quarto e quinto dedos da mão na população brasileira*
}

\section{Prevalence of Absence of Function of the Flexor Digitorum Superficialis Muscle Tendons in the Fourth and Fifth Fingers of the Hand in the Brazilian Population}

\author{
Bruno Macêdo Oliveira ${ }^{10}$ Carlos Henrique Fernandes ${ }^{10}$ Luis Renato Nakachima1잉 \\ João Baptista Gomes dos Santos ${ }^{10}$ Celso Kiyoshi Hirakawa1이 Flavio Faloppa1(0) \\ ${ }^{1}$ Departamento de Ortopedia e Traumatologia, Universidade Federal \\ de São Paulo, São Paulo, SP, Brasil \\ Rev Bras Ortop 2020;55(4):448-454. \\ Endereço para correspondência Bruno Macêdo Oliveira, MD, \\ Departamento de Ortopedia e Traumatologia, Universidade Federal \\ de São Paulo, Rua Borges Lagoa, 786, São Paulo, SP, Brasil \\ (e-mail: brunomacedooliveira@gmail.com).
}

\section{Resumo \\ Palavras-chave \\ - dedos \\ - deformidades congênitas da mão \\ - tendões \\ - síndromes de compressão nervosa \\ - estudos prospectivos}

Objetivos Avaliar a prevalência da ausência de função dos tendões do músculo flexor superficial do quarto e quinto dedos em uma população brasileira.

Métodos Estudo prospectivo foi realizado no departamento de ortopedia e traumatologia de um hospital universitário. O estudo foi desenvolvido no período de outubro de 2017 a abril 2018. A amostra foi formada por voluntários sem histórico de trauma, cirurgia ou qualquer afecção dos membros superiores. Foram incluídos neste estudo indivíduos de ambos os gêneros com idade igual ou superior a 18 anos. A avaliação da função do tendão do múculo flexor superficial do quarto e quinto dedos foi realizada por meio de testes clínicos por três examinadores independentes. Para análise dos dados foi utilizado o programa GraphPad Prism 5.0 (GraphPad Software, San Diego, CA, EUA).

Resultados Foram analisados 1.008 voluntários totalizando 2.016 mãos. A prevalência da ausência de função do tendão do músculo flexor superficial do quarto dedo foi de $0,56 \%$ na mão direita e $0,99 \%$ na mão esquerda. Em relação ao gênero, foi verificado na mão direita uma maior prevalência da ausência de função do flexor superficial do quarto dedo no gênero feminino quando comparado ao masculino $(p=0,0328)$. Na mão esquerda não foi observada diferença entre os gêneros $(p=0,7562)$. A ausência de função do músculo flexor superficial no quinto dedo foi de $34,53 \%$ na mão direita e de $30,06 \%$ na mão esquerda. Quando analisamos o quinto dedo em relação aos gêneros, foi verificado que a prevalência da ausência de função do músculo flexor superficial foi mais frequente no gênero feminino que no masculino, tanto na mão direita $(p=0.0001)$ como na mão esquerda $(p=0.0003)$. Conclusão Apesar de haver estudos realizados separadamente em diversas etnias, não havia na literatura dados referentes à prevalência da ausência de função do músculo flexor
Trabalho desenvolvido na disciplina de cirurgia da mão e membro superior do departamento de ortopedia e traumatologia da Universidade Federal de São Paulo, São Paulo, SP, Brasil. recebido

18 de Janeiro de 2019

aceito

15 de Agosto de 2019
DOI https://doi.org/

10.1055/s-0039-3402458. ISSN 0102-3616.
Copyright $\odot 2020$ by Sociedade Brasileira License terms de Ortopedia e Traumatologia. Published by Thieme Revinter Publicações Ltda, Rio de Janeiro, Brazil 


\section{Abstract}

\author{
Keywords \\ - fingers \\ - hand congenital \\ deformities \\ - tendons \\ - nervous \\ compression \\ syndromes \\ - prospective studies
}

superficial do quarto e quinto dedos da mão em uma população miscigenada como a brasileira.

Objectives To evaluate the prevalence of absence of flexor digitorum superficialis muscle tendons function of the fourth and fifth fingers of the hand in the Brazilian population

Methods Prospective study performed at the orthopedics and traumatology department of a university hospital. The study was conducted from October 2017 to April 2018. The sample consisted of volunteers with no history of upper limb trauma, surgery or any other condition. This study included both male and female individuals over 18 years old. The flexor digitorum superficialis muscle tendons of the fourth and fifth fingers were evaluated through clinical tests by three independent examiners. The data were analyzed using GraphPad Prism 5.0 (GraphPad Software, San Diego, CA, USA).

Results A total of 2,016 hands from 1,008 volunteers were analyzed. The prevalence of absence of flexor digitorum superficialis muscle tendon function of the fourth finger was $0.56 \%$ at the right hand and $0.99 \%$ at the left hand. At the right hand, this prevalence was higher in females compared to males $(p=0.0328)$. No difference between genders was noted in the left hand $(p=0.7562)$. The prevalence of absence of flexor digitorum superficialis muscle tendon function of the fifth finger was $34.53 \%$ in the right hand and $30.06 \%$ in the left hand. This prevalence was higher in females compared to males, both at the right hand $(p=0.0001)$ and the left hand $(p=0.0003)$. Conclusion Even though there are studies performed separately in different ethnic groups, there were no data in the literature regarding the prevalence of absence of flexor digitorum superficialis muscle tendons function of the fourth and fifth fingers of the hand in a multiethnic population such as the Brazilian one.

\section{Introdução}

O músculo flexor superficial dos dedos (MFSD) é o maior músculo do antebraço e o único da camada intermediária dos músculos do antebraço. ${ }^{1}$ O MFSD tem duas origens, uma úmero-ulnar e outra radial. Possui quatro tendões independentes que se dirigem aos dedos longos após atravessarem o túnel do carpo. ${ }^{2,3}$

O sistema constituído pelo MFSD e seus tendões realiza a flexão de múltiplas articulações, tais como as articulações do punho, intercarpais, carpometacarpianas, metacarpofalângeas e interfalângicas proximais. ${ }^{3-5}$

Encontramos na literatura a descrição de músculos duplicados e acessórios, além de músculos anormais nos membros superiores. As variações anatômicas no MFSD e nos seus tendões têm prevalências distintas nas diversas populações estudadas. $^{5-9}$

No sistema musculoesquelético, o MFSD é um dos músculos com mais variações anatômicas do corpo humano. Suas alterações funcionais podem acontecer devido à morfologia, origem, inserção e possíveis interconexões. Na população em geral, a ausência da função exclusiva do tendão do MFS do o quinto dedo é a variação mais comum. . $^{3,4}$

A ausência da função do tendão do MFSD não tem despertado atenção dos ortopedistas por não afetar a força e a destreza na mão quando usadas nas atividades cotidianas. ${ }^{1,3}$ Entretanto, a ausência de função pode induzir a erros de diagnóstico durante o exame clínico para avaliação da presença de lesões do tendão do músculo flexor superficial do quarto e quinto dedos após ferimentos. ${ }^{5}$ o cirurgião deve estar atento a essas variações para melhor interpretar os achados do exame físico assim como para indicar procedimentos cirúrgicos de reparação. ${ }^{10-12}$

Na literatura, a prevalência da ausência da função do MFS do quinto dedo apresenta uma variação de $0 \%$ na população da Índia a $30,77 \%$ na população britânica. ${ }^{3-5}$ Tais variações podem ser justificadas por fatores étnicos, geográficos e diferenças metodológicas. Até o presente momento ainda não foi relatada na literatura a prevalência da ausência de função dos flexores superficiais do quarto e quinto dedo. Neste contexto, o objetivo deste estudo foi avaliar a prevalência da ausência de função dos MFS do quarto e quinto dedos em uma população multiétnica no Brasil.

\section{Materiais e Metodos}

A presente pesquisa consiste de um estudo prospectivo, transversal e observacional realizado no departamento de ortopedia e traumatologia de um hospital universitário. 0 estudo foi desenvolvido no período de outubro de 2017 a abril 2018. A amostra foi formada por acompanhantes de pacientes que se tornaram voluntários. Esses voluntários não tinham histórico de trauma, cirurgia ou qualquer afecção nos membros superiores. Foram incluídos neste estudo 
indivíduos de ambos os gêneros que tivessem idade igual ou superior a 18 anos.

O projeto foi submetido e aprovado pela Plataforma Brasil e pelo comitê de ética sob o número 2481991, de acordo com as recomendações da resolução 466/96 do Conselho Nacional de Saúde para pesquisas em seres humanos. $O$ pesquisador responsável convidou os voluntários a participarem desta pesquisa e neste momento explicou as etapas do estudo. Para firmar a autorização e participação dos pacientes, o termo de consentimento livre e esclarecido (TCLE) foi entregue e assinado.

A identificação da função flexora superficial de cada dedo foi realizada por dois testes clínicos executados em ambas as mãos por três examinadores diferentes. Cada examinador realizou os testes descritos a seguir separadamente e em diferentes voluntários. Desta forma, um mesmo examinador não avaliou todos os voluntários, o que diminuiu o risco de viés de informação.

Para o quinto dedo, a identificação foi realizada mantendose todos os dedos em extensão com exceção do próprio quinto dedo. $O$ punho foi mantido em supinação completa e extensão neutra. O voluntário foi solicitado a flexionar o dedo mínimo. A presença da função do MFSD foi estabelecida sempre que houvesse a flexão da articulação interfalângica proximal (IFP) sem flexão da articulação interfalângica distal (IFD) (-Figura 1). A ausência da função do MFSD foi estabelecida quando a flexão da articulação IFP não fosse realizada ou quando a flexão da articulação IFP fosse realizada apenas em conjunto com a flexão da articulação IFD ( - Figura 2). 0 teste também foi considerado negativo quando a flexão da IFP do quinto dedo só fosse realizada em conjunto com a flexão da IFP do quarto dedo. Desta forma não havia função individual do MFS do quinto dedo.

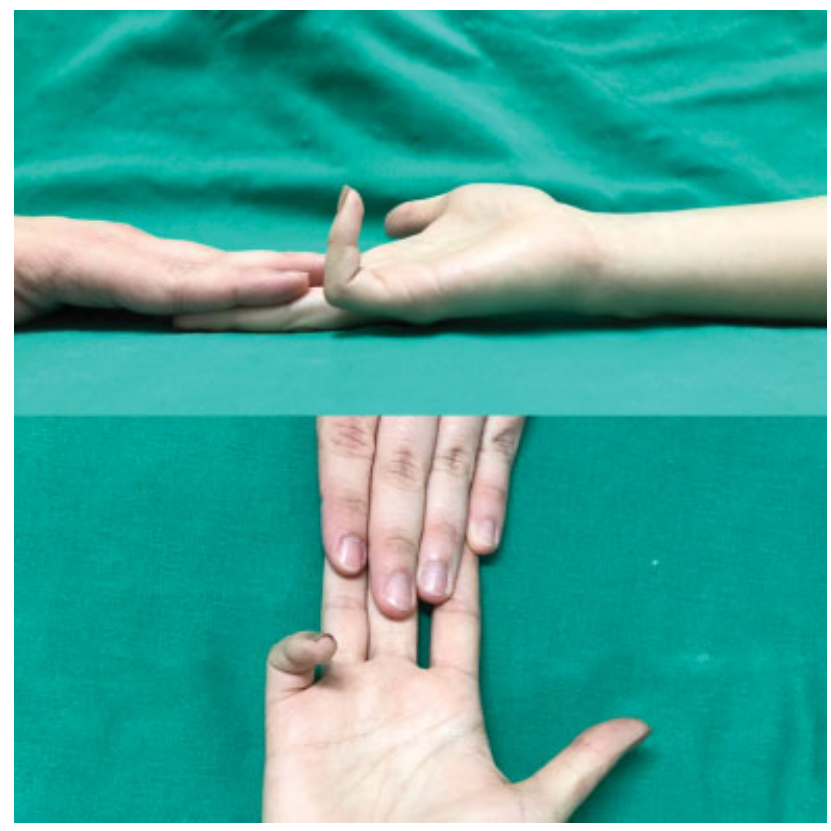

Fig. 1 Teste clínico para avaliar a presença da função do flexor superficial do quinto dedo. A flexão isolada da articulação interfalângica proximal confirma a função do músculo flexor superficial do quinto dedo.
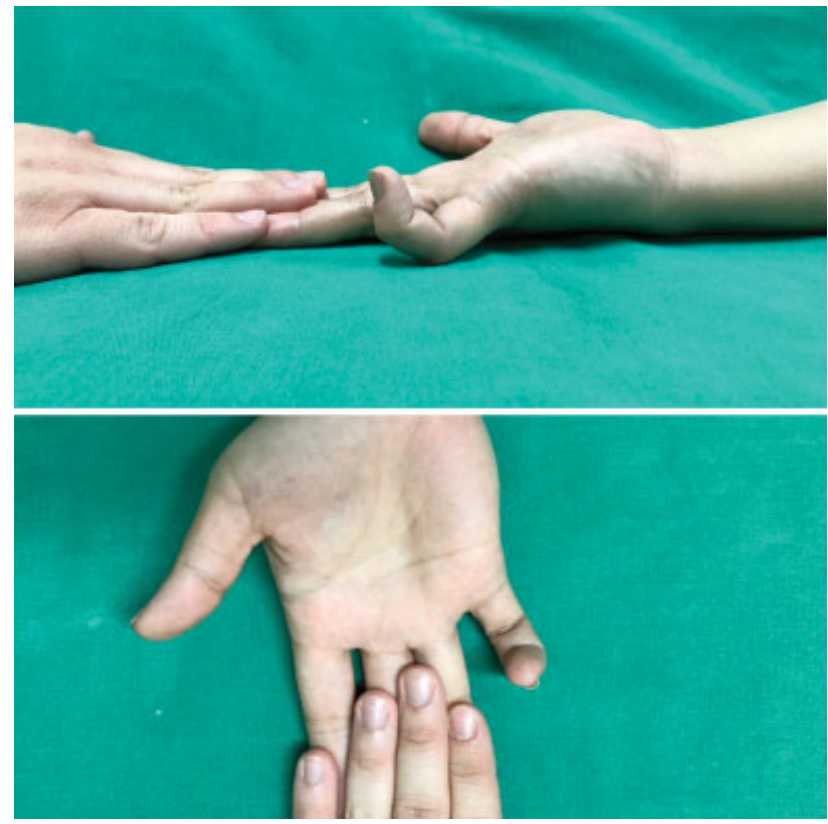

Fig. 2 Teste clínico para avaliar a presença da função do flexor superficial do quinto dedo considerado negativo.

Para o quarto dedo, a identificação foi realizada com todos os dedos estendidos exceto o quarto dedo. O punho foi mantido em supinação completa e extensão neutra. O voluntário foi solicitado a flexionar o quarto dedo. Se a flexão da articulação IFP ocorresse sem flexão da articulação IFD o teste foi interpretado como a presença da função do MFSD ( - Figura 3). Se a flexão da articulação IFP não ocorresse ou a flexão da articulação IFP ocorresse apenas em conjunto com a flexão da articulação IFD, era configurada ausência da função MFSD (-Figura 4).

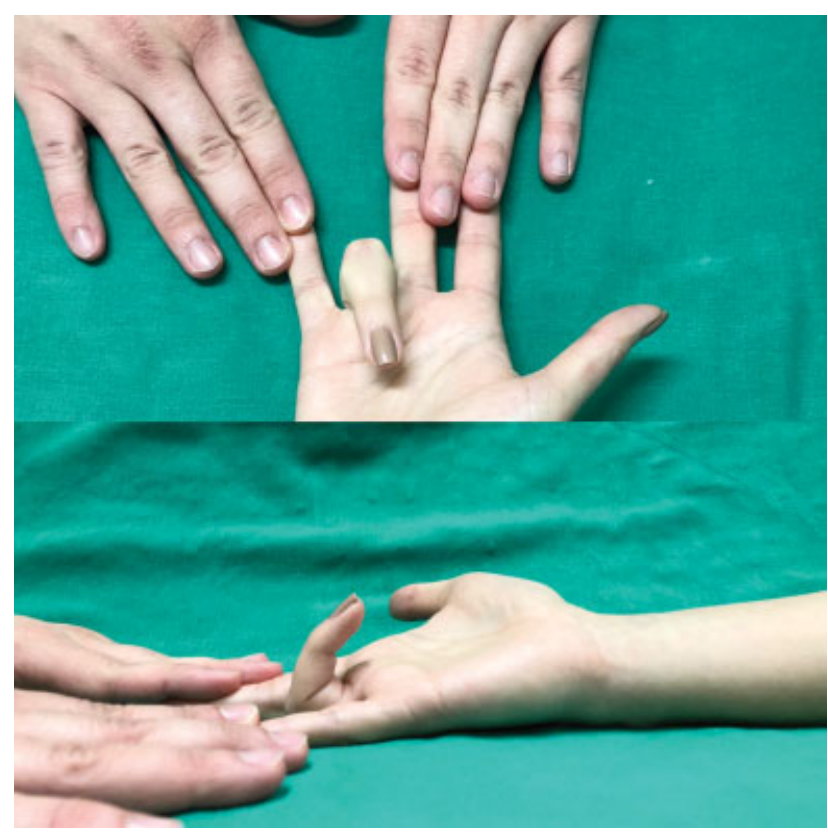

Fig. 3 Teste clínico para a identificação da presença da função flexora superficial do quarto dedo. 


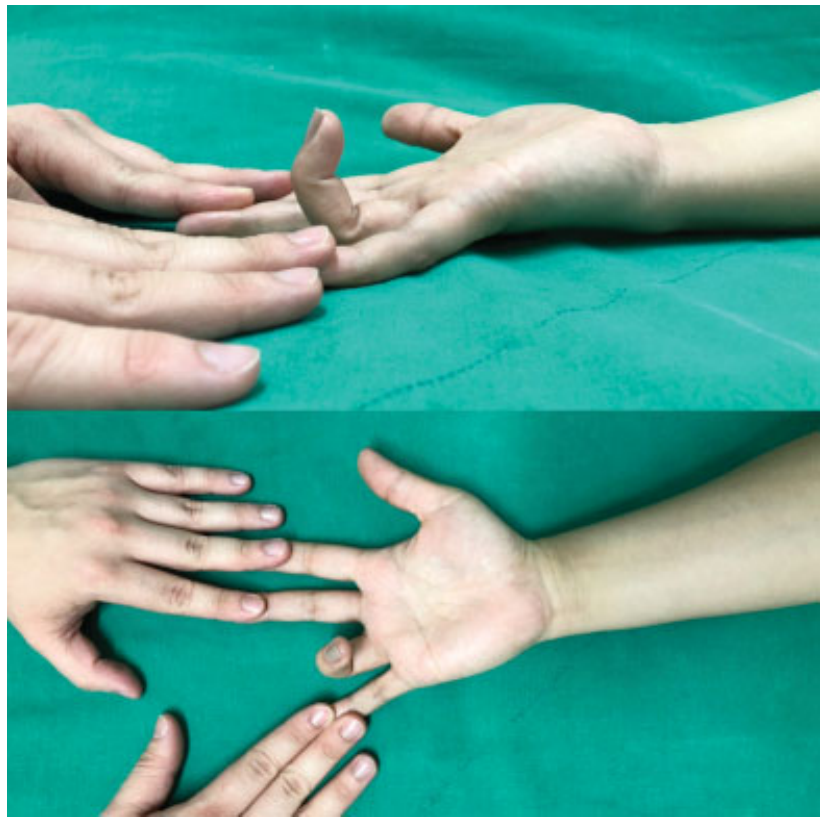

Fig. 4 Teste clínico para a identificação da presença da função flexora superficial do quarto dedo considerado negativo.

\section{Análise Estatística}

Todos os dados coletados foram organizados no software Excel (Microsoft Corp., Redmond, WA, EUA). A análise descritiva foi expressa em frequência e proporção. Para testar a homogeneidade entre as proporções foi utilizado o teste do qui-quadrado ou o teste exato de Fisher. Para análise dos dados foi utilizado o programa GraphPad Prism 5.0 (GraphPadSoftware, San Diego, CA, EUA), sendo adotado o nível de significância de $5 \%$.

\section{Resultados}

O presente estudo avaliou a prevalência da ausência de função dos tendões flexores superficiais do quarto e quinto dedos da mão em 1.008 indivíduos, totalizando 2.016 mãos.
Quanto ao gênero, 531 (52,67\%) pacientes eram do sexo masculino e 477 (47,33\%) do sexo feminino (-Figura 5). A idade variou de 18 a 70 anos com média de 38,42 anos.

A prevalência da ausência de função do tendão do MFS do quarto dedo da mão foi de $06(0,56 \%)$ na mão direita e de 10 $(0,99 \%)$ na mão esquerda ( - Figura 6). Essa diferença não foi significativa. De acordo com o gênero, foi verificado que na mão direita houve maior prevalência desta ausência no sexo feminino em relação ao sexo masculino $(p=0,0328)$. Já na mão esquerda não foi observada diferença entre os gêneros $(p=0,7562)$. Os números absolutos podem ser encontrados na - Tabela 1.

A prevalência da ausência de função do tendão flexor superficial do quinto dedo da mão foi analisada, sendo observada uma maior prevalência da ausência do MFS do quinto dedo na mão direita (34,53\%) que na esquerda (30,06\%), com significância estatística $(p=0,0361)$ ( - Figura 7). Quando analisamos a prevalência da ausência de função do tendão flexor superficial do o quinto dedo de acordo com o gênero, observamos que essa ausência foi mais frequente no sexo feminino que no masculino. Na mão direita observamos $37,10 \%$ no sexo feminino versus $23,72 \%$ no sexo masculino ( $p=0.0001)$. Já na mão esquerda, a prevalência da ausência de função do MFS no sexo feminino foi de $40,26 \%$ versus $29,38 \%$ no sexo masculino ( $p=0.0003)$. Os números absolutos podem ser encontrados na - Tabela 2.

\section{Discussão}

Estudos têm sido realizados nas diferentes regiões geográficas do mundo para elucidar a prevalência da ausência de função do MFS do quarto e quinto dedos. No entanto, existe uma escassez de dados em uma população formada por múltiplas etnias como a encontrada no Brasil.

O presente estudo analisou uma população com a idade média de 38 anos. Esse dado se aproxima do encontrado em outros estudos, que relataram a idade média de 40 e 41 anos. $^{13,14}$ Nosso estudo apresentou uma idade média

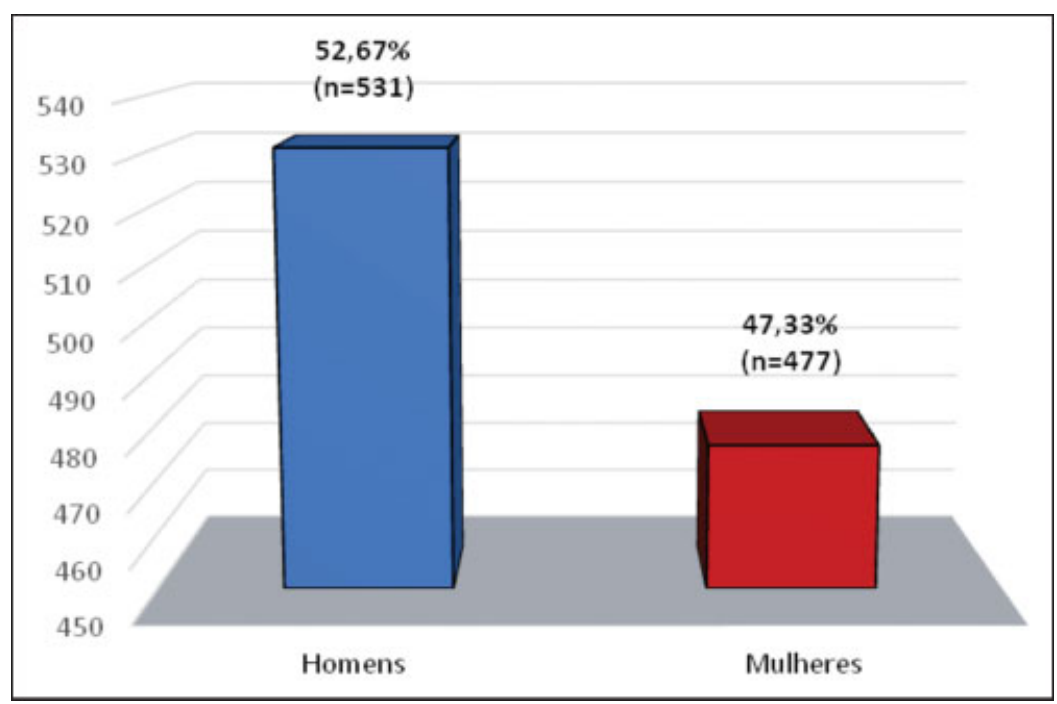

Fig. 5 Distribuição quanto ao gênero dos voluntários avaliados. 
452 Ausência de Função dos Tendões do Músculo Flexor Superficial do Quarto e Quinto Dedos Oliveira et al.

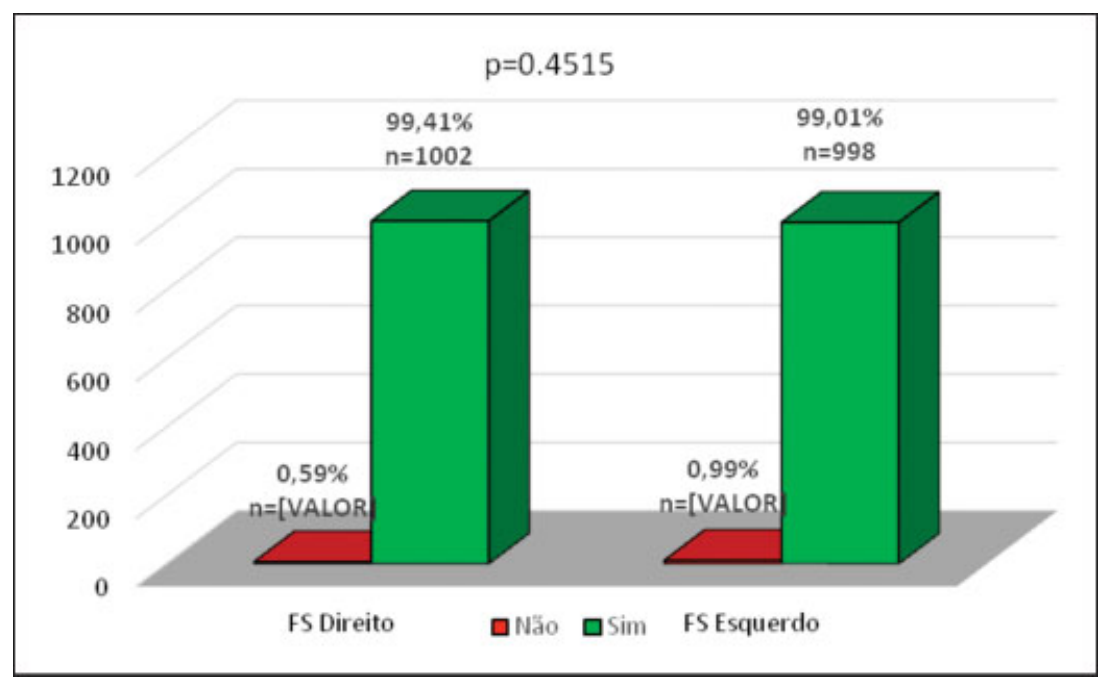

Fig. 6 Prevalência geral da presença dos tendões dos músculos flexores superficiais do quarto dedo da mão quanto ao lado.

Tabela 1 Prevalência da ausência de função dos tendões dos músculos flexores superficiais do quarto dedo da mão de acordo com o gênero

\begin{tabular}{|c|c|c|c|c|c|c|}
\hline \multirow[b]{2}{*}{ Gênero } & \multicolumn{3}{|l|}{ MFSD - Direito } & \multicolumn{3}{|l|}{ MFSD - Esquerdo } \\
\hline & $\begin{array}{l}\text { Prevalência } \\
\text { de Função } \\
\text { \% (n) }\end{array}$ & $\begin{array}{l}\text { Ausência } \\
\text { de Função } \\
\% \text { (n) }\end{array}$ & $p$ & $\begin{array}{l}\text { Prevalência } \\
\text { de Função } \\
\text { \% (n) }\end{array}$ & $\begin{array}{l}\text { Ausência } \\
\text { de Função } \\
\text { \% (n) }\end{array}$ & $p$ \\
\hline Homens & $1100 \%(n=471)$ & $0 \%(n=0)$ & 0,0328 & $98,88 \%(n=525)$ & $1,12 \%(n=6)$ & 0,7562 \\
\hline Mulheres & $98,88 \%(n=531)$ & $1,12 \%(n=6)$ & & $99,17 \%(n=473)$ & $0,83 \%(n=4)$ & \\
\hline
\end{tabular}

Abreviatura: MFSD, músculo flexor superficial dos dedos.

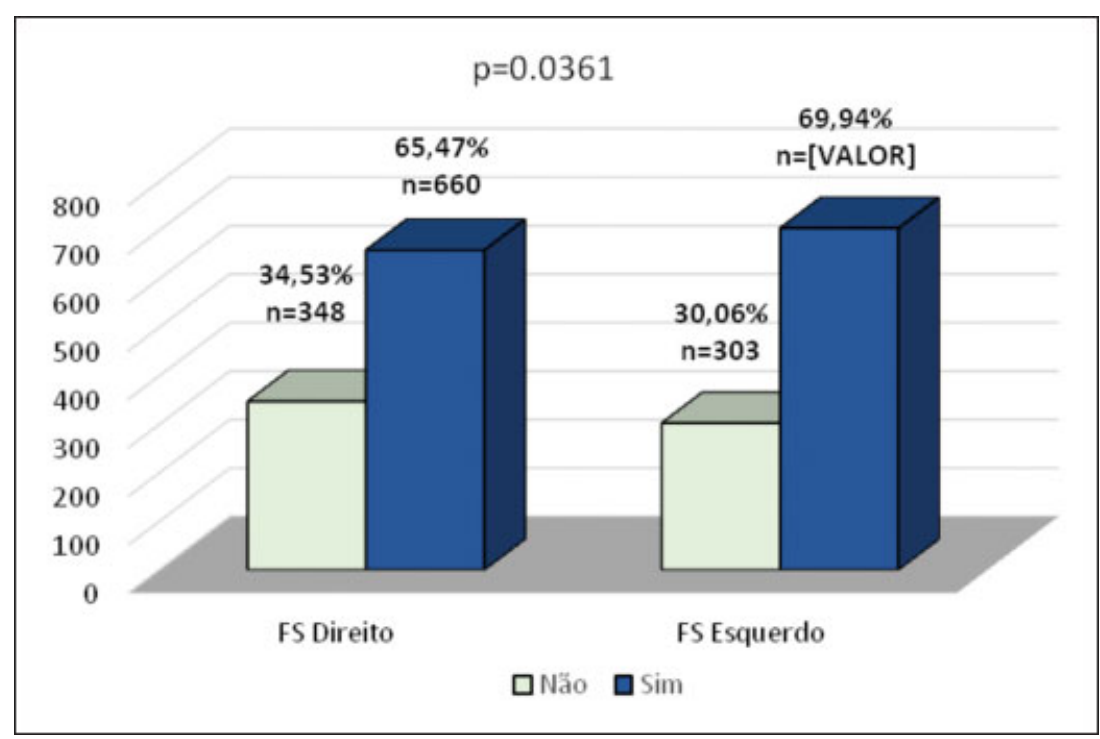

Fig. 7 Prevalência da ausência de função dos flexores superficiais do quinto dedo da mão.

superior à relatada nos estudos de Kigera e Katusiime, ${ }^{15}$ que analisaram uma população com idade média de 25 anos e de Raouf et al., ${ }^{16}$ que relataram a idade média de 23 anos.

As diferenças observadas entre os estudos podem ser justificadas principalmente devido ao grupo populacional estudado. Os estudos que analisaram a população em geral apresentaram um perfil de idade superior como a encontrada no nosso estudo. Em contrapartida, outros estudos analisaram grupos específicos, tais como estudantes, e, provavelmente devido a esse fato, apresentam uma faixa etária menor. A prevalência de ausência de função do MFS do quinto dedo pode afetar uma população ativa da sociedade 
Tabela 2 Prevalência da ausência de função dos tendões dos músculos flexores superficiais do quinto dedo da mão de acordo com o gênero

\begin{tabular}{|l|l|l|l|l|l|l|}
\hline & \multicolumn{2}{|l|}{ MFSD - Direito } & \multicolumn{2}{l|}{ MFSD - Esquerdo } \\
\hline Gênero & $\begin{array}{l}\text { Presença de Função } \\
\%(\mathbf{n})\end{array}$ & $\begin{array}{l}\text { Ausência de Função } \\
\%(\mathbf{n})\end{array}$ & $p$ & $\begin{array}{l}\text { Presença de Função } \\
\%(\mathbf{n})\end{array}$ & $\begin{array}{l}\text { Ausência de Função } \\
\%(\mathbf{n})\end{array}$ & $p$ \\
\hline Homens & $76,28 \%(n=405)$ & $23,72 \%(n=126)$ & 0.0001 & $70,62 \%(n=375)$ & $29,38 \%(n=156)$ & 0,0003 \\
\hline Mulheres & $62,90 \%(n=300)$ & $37,10 \%(n=177)$ & & $59,74 \%(n=285)$ & $40,26 \%(n=192)$ & \\
\hline
\end{tabular}

Abreviatura: MFSD, músculo flexor superficial dos dedos.

gerando impactos econômicos e comprometendo a carreira de grupos específicos e altamente especializados, tais como os músicos. ${ }^{13-16}$

Nossos resultados são concordantes com outros estudos que reportaram que os indivíduos do gênero feminino apresentaram com maior frequência a ausência de função do MFS do quinto dedo do que indivíduos do gênero masculino. Por outro lado, outros estudos mostraram que ambos os gêneros tinham prevalências semelhantes quanto à ausência de função do MFS do quarto e quinto dedos. Além disso, a prevalência no gênero feminino encontrada neste estudo em ambas as mãos foi maior que a encontrada no estudo de Bowman et al., que reportaram a prevalência de $18,6 \%$ na população feminina. ${ }^{9-15}$

Após avaliar nossos resultados e revisar a literatura, parece existir uma relação genética na prevalência baseada na etnia. Aqui nós observamos uma alta prevalência da ausência de função do MFS do quinto dedo de acordo com os relatos anteriores da literatura. Esses achados provavelmente são devidos à miscigenação encontrada no Brasil. Na África, a taxa global da prevalência da ausência de função foi baixa, chegando a 2,5\%. ${ }^{15}$ A prevalência da ausência de função do flexor superficial do quinto dedo relatadas nos países orientais foram mais baixas, tais como de $1,3 \%$ no Egito $^{16}$ e $4,3 \%$ no Iran. ${ }^{17}$ Entre a população turca a prevalência é $18,5 \%$. Já na China a prevalência da ausência foi de $6,4 \%{ }^{18}$ Nos EUA, esta variou de 16 a 21\%. Enquanto que, na Inglaterra, a prevalência de ausência da função FSD do quinto dedo na população geral varia de 13,7 a $25 \%,{ }^{19}$ e em músicos esta foi de $3,3 \%$.

Outra diferença entre os estudos inclui o número amostral abordado em cada relato, que varia de 50 pessoas no estudo pioneiro de 1989 nos EUA a 800 pessoas no estudo africano. Dessa forma, é questionável se os dados relatados refletem a real prevalência da ausência do tendão do FSD do quinto dedo.

$O$ presente estudo foi o primeiro a reportar a prevalência da ausência de função dos flexores superficiais do quarto e quinto dedos na população brasileira. Obviamente, existem outras diferenças que justificam a alta prevalência encontrada além do número amostral. Estudos anteriores ${ }^{13-18}$ avaliaram populações relativamente uniformes do ponto de vista racial. A miscigenação encontrada no Brasil, e a presença do índio brasileiro podem ser fatores atuantes no aumento da prevalência da ausência de função dos flexores superficiais do quarto e quinto dedos da mão visto no presente estudo. Em nenhum dos trabalhos realizados em outras populações havia a presença do índio brasileiro nem suas interações genéticas com outras raças.
Uma recente metanálise analisou a prevalência da ausência de função do flexor do quinto dedo em diferentes estudos e sugeriu que existe uma tendência evolutiva produzida pela adaptação natural de gerar a independência do quinto dedo. Este fator provavelmente reduzirá a prevalência da ausência de função deste músculo nas populações.

Os pacientes frequentemente se apresentam ao departamento de emergência com lesões agudas na mão e no antebraço. 0 cuidado padrão destes pacientes inclui o exame completo das funções neurológicas e vasculares. No entanto, tem sido demonstrado que o tendão flexor superficial do quinto dedo pode estar funcionalmente ausente, e a simples avaliação clínica da presença deste tendão deve ser incluída na rotina de cuidados, ${ }^{20-22}$ especialmente na população brasileira, na qual constatamos uma alta prevalência da ausência de função desse flexor. Assim, a identificação da prevalência da ausência da função do MFSD pode ser útil para cirurgião da mão como parte dos critérios para reparo cirúrgico de afecções tendinosas.

\section{Conclusão}

Apesar de haver estudos realizados separadamente em diversas etnias, não havia na literatura dados referentes à prevalência da ausência de função do MFS do quarto e quinto dedos da mão em uma população miscigenada como a brasileira. Lesões desses tendões devem ser corretamente avaliadas pois atingem a parcela produtiva da população, e sequelas oriundas de erro diagnostico podem produzir sérios impactos econômicos. A decisão de exploração e reparo cirúrgico deve ter como base o adequado exame físico e o conhecimento da alta prevalência de ausência da função desses tendões, principalmente do MFS do quinto dedo da mão, como evidenciado no presente estudo.

Conflito de Interesses

Os autores declaram não haver conflito de interesses.

\section{Referencias}

1 Caetano EB, Sabongi Neto JJ, Ribas LAA, Milanello EV. Accessory muscle of the flexor digitorum superficialis and its clinical implications. Rev Bras Ortop 2017;52(06):731-734

2 Bernardes A, Melo C, Pinheiro S. A combined variation of Palmaris longus and Flexor digitorum superficialis: Case report and review of literature. Morphologie 2016;100(331):245-249

3 Yammine K, Erić M. Agénésie, déficit fonctionnel, et variations anatomiques les plus courantes du flexor digitorum superficialis 
454 Ausência de Função dos Tendões do Músculo Flexor Superficial do Quarto e Quinto Dedos Oliveira et al.

de l'auriculaire: une méta-analyse. Hand Surg Rehabil 2018;37 (02):77-85

4 Gupta A, Kumar V. Bilateral Absence of Flexor Digitorum Superficialis (FDS) Tendon of the Little Finger: Clinical Significance. J Clin Diagn Res 2014;8(02):135-136

5 Tejaswi HL, Shilpashree YD. Clinical assessment of agenesis of palmaris longus and flexor digitorum superficial is in Indian population. Int J Rec Trends Sci Technol 2014;10(03):492-494

6 Yilmaz A, Bozer C, Uluçam E, Taşkinalp O. An anomaly of flexor muscles of the fifth (little) finger of the hand: an anatomical case report. Clinics (São Paulo) 2009;64(11):1133-1135

7 Ndibazza J, Lule S, Nampijja M, et al. A description of congenital anomalies among infants in Entebbe, Uganda. Birth Defects Res A Clin Mol Teratol 2011;91(09):857-861

8 Elliot D, Khandwala AR, Kulkarni M. Anomalies of the flexor digitorum superficialis muscle. J Hand Surg $\mathrm{Br}$ 1999;24(05): 570-574

9 Bowman P, Johnson L, Chiapetta A, Mitchell A, Belusko E. The clinical impact of the presence or absence of the fifth finger flexor digitorum superficialis on grip strength. J Hand Ther 2003;16 (03):245-248

10 Guler F, Kose O, Turan A, Baz AB, Akalın S. The prevalence of functional absence of flexor digitorum superficialis to the little finger: a study in a Turkish population. J Plast Surg Hand Surg 2013;47(03):224-227

11 Tan JS, Oh L, Louis DS. Variations of the flexor digitorum superficialis as determined by an expanded clinical examination. J Hand Surg Am 2009;34(05):900-906

12 Yesilada AK, Tatlıdede HS, Çakmak E, Kıyak MV, Kılınç L. Anomalous large unique muscle belly of flexor digitorum superficialis and the absence of palmaris longus in the forearm. J Plast Reconstr Aesthet Surg 2013;66(01):137-139

13 Godwin Y, Wheble GA, Feig C. Assessment of the presence of independent flexor digitorum superficialis function in the small fingers of professional string players: is this an example of natural selection? J Hand Surg Eur Vol 2014;39(01):93-100

14 Puhaindran ME, Sebastin SJ, Lim AY, Xu WX, Chen YM. Absence of flexor digitorum superficialis tendon in the little finger is not associated with decreased grip strength. J Hand Surg Eur Vol 2008;33(02):205-207

15 Kigera JW, Katusiime A. Prevalence of agenesis of flexor digitorum superficialis of the fifth digit in East Africa through clinical examination. SA Orthop J 2011;10(04):75-77

16 Raouf HA, Kader GA, Jaradat A, Dharap A, Fadel R, Salem AH. Frequency of palmaris longus absence and its association with other anatomical variations in the Egyptian population. Clin Anat 2013;26(05):572-577

17 Enhesari A, Saied A, Mohammadpoor L, Ayatollahi Mousavi A Arabnejhad F. Presence or absence of palmaris longus and fifth superficial flexor digitorum; is there any effect on median nerve surface area in wrist sonography. Iran J Radiol 2014;11(04): e14441

18 Sebastin SJ, Lim AY. Clinical assessment of absence of the palmaris longus and its association with other anatomical anomalies- a Chinese population study. Ann Acad Med Singapore 2006;35(04): 249-253

19 Townley WA, Swan MC, Dunn RL. Congenital absence of flexor digitorum superficialis: implications for assessment of little finger lacerations. J Hand Surg Eur Vol 2010;35(05):417-418

20 Wilcox DC, Buschbacher R, Bergeron J. Incidence of the inability to flex the proximal interphalangeal joint in normal subjects. Arch Phys Med Rehabil 1998;79(11):1405-1407

21 Austin GJ, Leslie BM, Ruby LK. Variations of the flexor digitorum superficialis of the small finger. J Hand Surg Am 1989;14(2 Pt 1):262-267

22 Baker DS, Gaul JS Jr, Williams VK, Graves M. The little finger superficialis-clinical investigation of its anatomic and functional shortcomings. J Hand Surg Am 1981;6(04):374-378 